\title{
Technical Evaluation of the Carolo-Cup 2014 - A Competition for Self-Driving Miniature Cars
}

\author{
Sebastian Zug, Christoph Steup, \\ Julian-B. Scholle \\ Otto-von-Guericke Universität Magdeburg \\ Department for Distributed Systems \\ 39106 Magdeburg, Germany \\ Email: \{zug, steup\}@ovgu.de, \\ jscholle@st.ovgu.de
}

\author{
Christian Berger, Olaf Landsiedel \\ Chalmers University of Technology \& \\ University of Gothenburg \\ Department of Computer Science and Engineering \\ 41296 Göteborg, Sweden \\ Email: \{christian.berger,olafl $\} @$ chalmers.se
}

\author{
Fabian Schuldt, Jens Rieken, \\ Richard Matthaei, Thomas Form \\ Technische Universität Braunschweig \\ Department Vehicle Electronics \\ 38106 Braunschweig, Germany \\ Email: \{schuldt,rieken, \\ matthaei,form $\} @$ ifr.ing.tu-bs.de
}

\begin{abstract}
The Carolo-Cup competition conducted for the eighth time this year, is an international student competition focusing on autonomous driving scenarios implemented on 1:10 scale car models. Three practical sub-competitions have to be realized in this context and represent a complex, interdisciplinary challenge. Hence, students have to cope with all core topics like mechanical development, electronic design, and programming as addressed usually by robotic applications.

In this paper we introduce the competition challenges in detail and evaluate the results of all 13 participating teams from the 2014 competition. For this purpose, we analyze technical as well as non-technical configurations of each student group and derive best practices, lessons learned, and criteria as a precondition for a successful participation. Due to the comprehensive orientation of the Carolo-Cup, this knowledge can be applied on comparable projects and related competitions as well.
\end{abstract}

\section{INTRODUCTION}

Since decades student competitions in engineering disciplines represent an important part of university education [1]. Challenging tasks, inspiring team work, and the competitive character attract students. Additionally, the orientation towards a competition in conjunction with an increased visibility by public interest boost the motivation of the students. In this context, practical and theoretical knowledge can be combined and increased on a high level. Besides an improvement of technical skills, every team-oriented competition generates the atmosphere of a "realistic" development process. In this regard, students have to organize themselves related to milestones, interfaces, system integration, testing, and responsibilities in order to reach the goal. Based on these experiences, the importance of software engineering, project management, and so forth become clear. Additionally, student competitions in logistic robotics [2], autonomous car [3], vessel or submarine control offer the sole opportunity to develop skills in interdisciplinary projects addressing mechanical and electrical engineers as well as computer scientists [4]. The combination of all these elements improves significantly the engineering education. An interesting historical overview is given by Bräunl [5] related to this fact.

Carolo-Cup ${ }^{1}$ is one of these competitions organized by the Technische Universität Braunschweig (Germany) every year. The competition focuses on implementing small scale self-driving cars in different scenarios. Sec. II presents details of the current competition while [6] gives a historical overview. Since all authors either supervise one or more teams or are involved in the competition's organization, in depth knowledge of the competition as well as typical problems of the teams are included and presented in this work.

${ }^{1}$ http://www.carolo-cup.de

\section{A. Problem Domain}

While such competitions are exciting and motivating for participants, the contestants are also facing various challenges during the realization of their solutions. Participants from the Carolo-Cup mentioned several reasons for unwanted or unexpected behavior as listed in the following:

- unstable behavior of the developed algorithms,

- problems to meet the specific properties of the arena, or

- physical hardware crashes due to transport or wrong handling without a chance of repair.

This collection addresses challenges well known for every engineer and observable in many applications working with autonomous or robotic scenarios. They combine technical and maintenance failures as well as organizational and management pitfalls. The student teams are confronted with all of them. However, unlike experienced engineers, they usually do not pay attention to appropriate prevention strategies. Flexible debug tools or parameter adaptations as well as model driven verification are not even considered by many teams [7]. This lack of awareness should be overcome by a positive learning experience while working as part of a Carolo-Cup team.

\section{B. Research Goal and Contributions}

However, there is a danger that these goals will not be achieved due to faulty but fundamental strategic decisions. The team composition, hardware configuration, or the choice of a certain software library often threatens the complete project.

Therefore, our goals in this paper are:

1) To provide guidance on best practices to new teams to ease their first participation in the CaroloCup,

2) To support existing teams in reviewing their setup and approach by providing a comprehensive overview and performance analysis of all teams from 2014 and

3) To reach out to the academic community to collect feedback on the approaches and potentially identified improvements.

For this purpose we analyzed the presentations given by each team and identified their hardware structure, software concepts, and solution strategies pursuing the following questions:

RQ-1: What typical issues were common in all teams?

$R Q-2:$ Which typical hardware and software strategies were successful?

$R Q-3:$ What are the main pitfalls for new teams?

The identification of these corner stones is not only applicable on Carolo-Cup teams. Due to the general focus of the discipline, they are helpful for comparable projects as well. In this regard, the 
paper also initializes an exchange of methods and strategies between different Carolo-Cup teams. Related to the newest developments in automotive safety functions (ESP, ABS, ASR) addressed by some teams, our findings are of interest for a larger scientific community as well.

\section{Structure of the Article}

The paper is structured as follows: The next section introduces the Carolo-Cup and describes the different challenges. Sec. III evaluates the team concepts related to the achieved results and investigates the challenge of the parking task in detail. The basic lessons based on this analysis are combined with the individual experiences of each team in Sec. IV.

\section{The CARolo-Cup Competition}

In the following, we describe the annual student competition Carolo-Cup. This competition for self-driving miniature vehicles in 1:10 scale, as shown in Fig. 1, was introduced at Technische Universität Braunschweig in 2008. One of the motivational drivers was the successful participation in the 2007 DARPA Urban Challenge [8] for real-scale self-driving cars on the one hand, and the need to involve students from engineering disciplines already in their education with the challenges of this technology on the other hand.

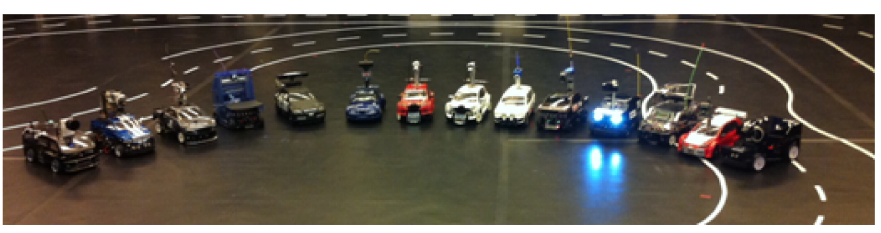

Figure 1. Contestants in the Carolo-Cup 2014 competition.

This aspect is getting more and more apparent as important automotive original equipment manufacturers (OEMs) have announced to make self-driving vehicular technology and ready for the market [9]. In this regard, the competition clearly adds a benefit to participating students as they have to work on a practical level for a given, nonextensible time-frame with hard deadlines and finally to deliver a product according to the official rules and regulations document.

\section{A. Tasks in the Competition}

a) General Setup: The competition takes place in conjunction with an industrial and scientific symposium on automation, assistance, and embedded systems for mobility solutions in early February every year. The competition opens for practicing on the test track usually on Sunday and Monday; the actual competition is taking place on Tuesday. While the first two days allow the student groups to accommodate with the local conditions like lightening and unexpected interferences in the perceived sensor data, the actual competition day starts with a presentation of the vehicle's concept in front of a jury consisting of industrial practitioners. In the evening, the actual dynamic driving competitions are taking place.

b) Presentation of the Concept: The participants need to present the concepts of their solution to a jury consisting of industrial practitioners and researchers. The presentation needs to cover the following aspects: (a) hardware architecture, (b) software architecture, (c) energy consumption, (d) costs, (e) perception and control for lanefollowing, (f) perception and control for overtaking and intersection handling, and $(\mathrm{g})$ perception and control for parking.

Giving a good presentation is utterly important as the amount of points that a team could get for this part can provide up to $35 \%$ of a maximum of 1000 points for the entire competition. In the last edition, the top team received 306 out of 350 points, while the team with the lowest points received only 181 ; on average, the teams received 243 points with a variance of 42 points.

c) Lane Following: The first dynamic discipline is to follow white lane markings on a black carpet as shown in Fig. 2. The track comprises of straight roads, arc-based curves, S-shaped curves, clothoid-based curves, intersections, and a parking strip.

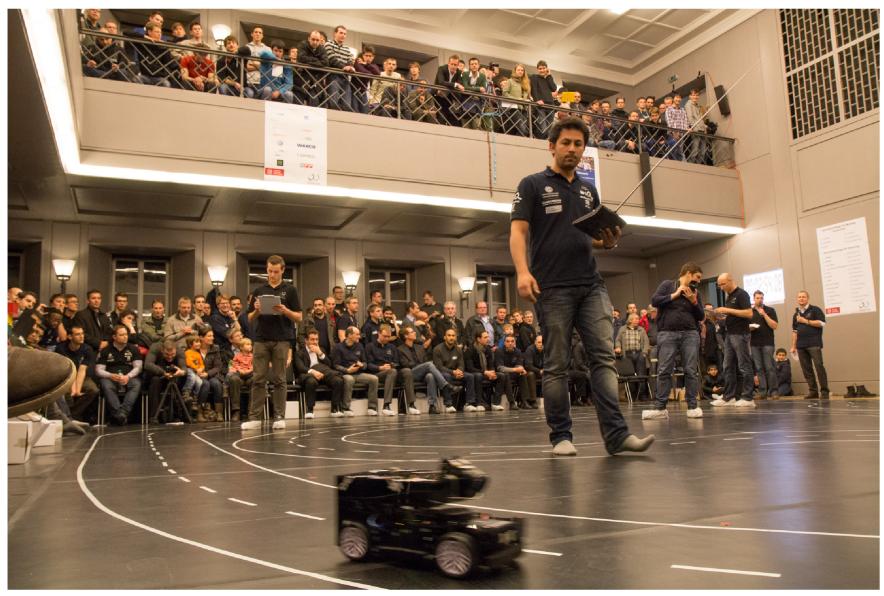

Figure 2. Driving in the discipline lane following.

However, the specific challenge is that some lane markings can be missing up to $1 \mathrm{~m}$ in length. Thus, the students' solutions need to compensate these missing parts by using appropriate algorithmic solutions to process the perceived sensor data. Penalty points are rewarded when the car is leaving the own lane.

The winning team got the maximum number of points for this task (200 points); the top three participants got 191 points on average with a variance of 9 points. In this regard, these three teams had a similar performance in this task.

d) Lane-Following and Overtaking Obstacles: The second task for the miniature cars is similar to the previous challenge. However, the aforementioned task focused purely on the top-speed in terms of driving as many rounds as possible within a 180s time limit without stopping at intersections. As an extension, the cars now need to handle stationary and dynamic obstacles on various parts of the track. Additionally, the cars had to detect right-of-way situations at intersections and behave correctly without touching other obstacles or vehicles. The obstacles in this task are made of white paper boxes with predefined minimum dimensions.

Penalty points are rewarded when the car is touching any obstacle, not behaving correctly at intersections, or leaving the own lane except for overtaking maneuvers.

In this task, only three teams participated. The top team got the maximum number of points for this task ( 250 points). The average number of points in this task is 169 with a variance of 70 points. Thus, it is apparent that this task is rather challenging and not all teams are able to realize this vehicle function in a robust and reliable manner.

e) Parking on a Sideways Parking Strip: The last driving task is autonomous parking on a sideways parking strip next to a straight road. The self-driving miniature car is placed initially behind the start line and needs to find a parking spot between several obstacles that are realized by white paper boxes. The goal for this task is to park as fast as possible while choosing the smallest gap out of three possible 
ones $(0.55 \mathrm{~m}, 0.63 \mathrm{~m}$, and $0.7 \mathrm{~m})$ to get the highest points. Every team has three attempts and the resulting points of this task are the mean value of the three attempts.

Penalty points are rewarded when the self-driving miniature car is touching the paper boxes, is not placed in parallel to the straight road, touching the white lane marking after finishing the parking maneuver, or missing a safety margin to the front and rear boxes. Additional penalty points are rewarded if the car does not choose the smallest parking gap.

Six teams participated in the parking task for which the top team got 133 points out of 200 . The average number of points is 89 points with a variance of 29 points.

\section{B. Results of the 2014 Carolo-Cup}

Tab. I summarizes the results of the 2014 competition related to all four aforementioned disciplines. Just one out of 13 teams competed in all disciplines while four teams did not achieve a single point in the practical part.

Table I

RESULTS OF THE CAROLO-CUP 2014 DIVIDED TO THE FOUR DISCIPLINES, FOR FURTHER INVESTIGATION THE TEAMS ARE CLUSTERED IN THREE GROUPS (GREEN, BLUE, YELLOW)

\begin{tabular}{|c|c|c|c|c|c|c|}
\hline Team name & University & 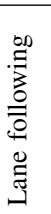 & 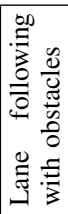 & 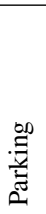 & 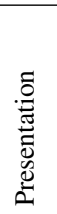 & 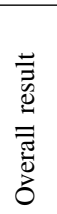 \\
\hline Spatzenhirn & Ulm & 191 & 250 & 133 & 290 & 864 \\
\hline CDLC & Braunschweig & 200 & 126 & & 304 & 630 \\
\hline TUM & Munich & 182 & 131 & & 306 & 619 \\
\hline Ostfalia-Cup & Wolfenbüttel & 72 & & 107 & 264 & 443 \\
\hline FAUST & Hamburg & 77 & & 7 & 259 & 412 \\
\hline Team THM & Gießen & 79 & & 53 & 259 & 390 \\
\hline e.Wölfe & Wolfsburg & 73 & & 98 & 192 & 362 \\
\hline S.A.D.I & Zwickau & 22 & & 67 & 247 & 335 \\
\hline Legendary & Gothenburg ${ }^{2}$ & 18 & & & 229 & 247 \\
\hline Tetrix & Gothenburg & & & & 218 & 218 \\
\hline GalaXis & Aachen & & & & 213 & 213 \\
\hline Berlin United & Berlin & & & & 196 & 196 \\
\hline Lippe Coast & Lippstadt & & & & 181 & 181 \\
\hline
\end{tabular}

The lane-following with obstacles contest was only tackled by the teams from Ulm, Braunschweig, and Munich. These teams are aggregated in the Green group. All other contestants are classified to the Blue and Yellow group depending on the number of successfully completed disciplines. The three classes are are used for further analysis in Sec. III.

\section{EVALUATION}

The comparison of competition results is straight forward as we can identify winning teams and less successful groups based on their rankings. However, to better analyze their final results, we define a set of categories to compare the teams on an abstract level.

\section{A. Data Basis}

The following analysis is founded on three information sources:

Team presentations are part of the competition and have to include a set of a mandatory elements as described in Sec. II. The presentations are available for

${ }^{2}$ Chalmers University, Sweden all participating teams as well as their video recordings.

Websites are used by many teams to publish news, present the team, find new team members, and to acknowledge their sponsors.

Registration forms of the Carolo-Cup ask for team member information and software libraries in addition to organizational questions.

However, the sample size of our data base is small and we have no assumptions related to the basic population. Consequently, this paper cannot provide a specific relation between team parameters and placement on a reasonable confidence level. It is rather our idea to detect the requirements of a successful participation in general. For this purpose, we organize the data in the mentioned three groups - Green, Blue, and Yellow.

\section{B. Technical Implementation vs. Results}

We discuss five aspects of the teams in this section: the nontechnical team configuration, costs, the sensors in use, the software structure, and the hardware selection. As an additional parameter we evaluated the presentations in order to recognize the focus of the team.

f) Team composition: 121 students took part in the Carolo-Cup 2014. Most of them are computer science students (62\%). The proportion of female students is very low compared to the general number of female engineering students at German Universities $\left(21 \%{ }^{3}\right)$.

Table II

TEAM COMPOSITIONS OF THE CAROLO-CUP 2014

\begin{tabular}{|c|c|c|c|c|c|c|}
\hline Team name & 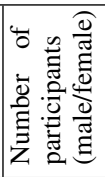 & 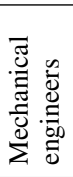 & 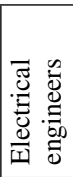 & 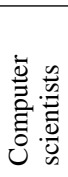 & $\stackrel{\bar{\Xi}}{\overline{0}}$ & 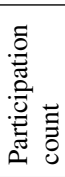 \\
\hline $\begin{array}{l}\text { Spatzenhirn } \\
\text { CDLC } \\
\text { TUM }\end{array}$ & $\begin{array}{l}12 \text { / } 0 \\
15 / 1 \\
14 / 2\end{array}$ & $\begin{array}{l}1 \\
8\end{array}$ & $\begin{array}{l}3 \\
8\end{array}$ & $\begin{array}{l}7 \\
7 \\
5\end{array}$ & 2 & $\begin{array}{l}5 \\
6 \\
5\end{array}$ \\
\hline $\begin{array}{l}\text { Ostfalia-Cup } \\
\text { FAUST } \\
\text { Team THM } \\
\text { e.Wölfe } \\
\text { S.A.D.I }\end{array}$ & $\begin{array}{r}10 / 0 \\
9 / 0 \\
8 / 0 \\
7 / 0 \\
9 / 0\end{array}$ & 2 & $\begin{array}{l}4 \\
5\end{array}$ & $\begin{array}{l}10 \\
9 \\
1 \\
6 \\
4\end{array}$ & $\begin{array}{l}1 \\
1\end{array}$ & $\begin{array}{l}4 \\
7 \\
2 \\
1 \\
6\end{array}$ \\
\hline $\begin{array}{l}\text { Legendary } \\
\text { Tetrix } \\
\text { GalaXis } \\
\text { Berlin United } \\
\text { Lippe Coast }\end{array}$ & $\begin{array}{l}5 / 0 \\
8 / 1 \\
9 / 0 \\
8 / 0 \\
3 / 0\end{array}$ & 2 & 2 & $\begin{array}{l}5 \\
9 \\
5 \\
8\end{array}$ & 1 & $\begin{array}{l}1 \\
1 \\
6 \\
3 \\
1\end{array}$ \\
\hline$\Sigma$ & $117 / 4$ & 15 & 22 & 76 & 8 & \\
\hline
\end{tabular}

Beside the general aspects, we analyzed the team configurations related to different aspects. Fig. 3a shows the team size and the achieved number of points without the presentation discipline. The teams belonging to Green have 12 to 16 participants, the other teams are significantly smaller.

The number of previous participations is visualized in Fig. $3 b$ for each team. An interesting result for new teams is visible: it does not exist a correlation between participation count and achieved points. Group Blue for instance covers first time participants and veterans of the Carolo-Cup. Obviously, teams are not able to transfer knowledge and expertise from a current competition to the next one. A possible reason might be the large fluctuation inside the teams.

${ }^{3}$ German Federal Statistical Office (2013) 


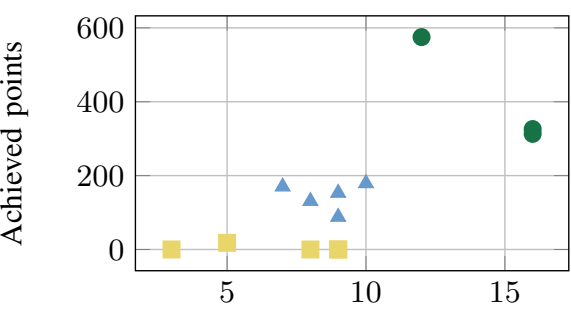

(a) Number of team members

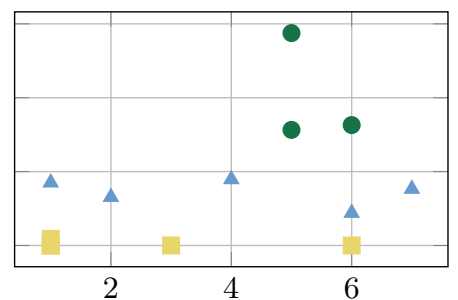

(b) Participation count

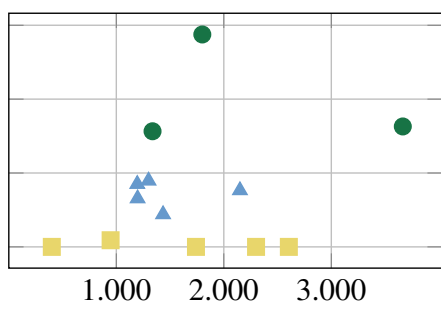

(c) Hardware costs

Figure 3. Analysis of team configuration and hardware costs related to the achieved points in practical disciplines.

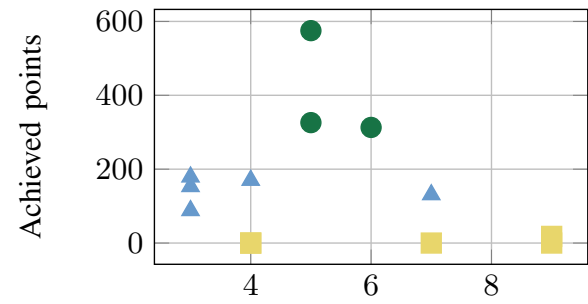

(a) Number of different sensor types

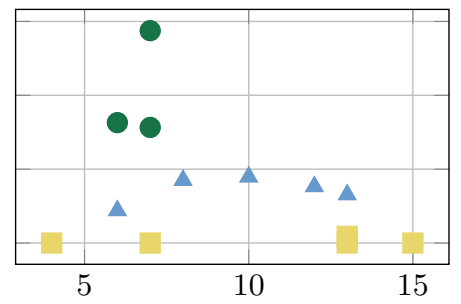

(b) Number of assembled sensors

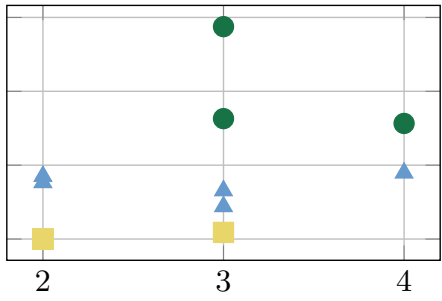

(c) Number of controllers

Figure 4. Analysis of sensor and hardware configuration related to the achieved points in practical disciplines (Green $\bullet$, Blue $\Delta$, Yellow $\square)$

While comparing current team lists with previous one, we recognized that just a minority of participants is involved more than one time.

The Carolo-Cup disciplines requests experts in three core disciplines: mechanical and electrical engineering as well as computer science. We structured the students of all teams accordingly. A benefit of interdisciplinary teams compared to mono-disciplinary ones cannot be recognized based on the available data sets.

The mentioned basic aspects represent a small selection of the complex processes inside a development team.

g) Hardware costs: All teams presented the financial background of their project. The hardware for an individual car model costs between 400 and 3,700€. Based on the illustration of Fig. 3c we suspect a "magic" border of at least $1,000 €$ that have to be invested for a competitive car. The composition of the costs is dominated by the sensor concept and the controller board in use. The team which constructed the most expensive car has integrated a laser scanner that ties up one third of the general costs.

h) Sensor setup: Suitable perception represents the most important challenge for all Carolo-Cup disciplines. The sensors have to detect the lane course, relative car position and orientation as well as obstacles; a comprehensive analysis of sensors and associated algorithms is presented in [6]. The results of our evaluation related to the sensor configuration are available in Fig. $4 \mathrm{a}, 4 \mathrm{~b}$ and Tab. III. The latter shows the number of teams per group applying a specific sensor type by empty and filled dots.

The top teams integrated a limited number of sensors in their cars compared to the less successful ones. Teams from the group Green use 5 to 6 sensor types and 6 to 7 sensor systems as visible in Fig. $4 \mathrm{a}$ and Fig. 4b. We suspect that a balanced number of sensor elements is a key issue of an successful participation. Obviously, teams from the Blue and Yellow decided to integrate too many or too few sensors.

Tab. III summarizes the the sensor configurations on an abstract level. All teams applied a camera system reaching from low budget webcams up to industrial camera systems. It is notable, that 2 out of 3 teams from group Green implemented a mono-chrome camera. Accordingly, both teams emphasized the need for a high
Table III

COMPARISON OF THE SENSOR SETUP.

\begin{tabular}{|c|c|c|c|}
\hline & \multicolumn{3}{|c|}{ Group } \\
\hline Parameter & Green & Blue & Yellow \\
\hline Camera & $\bullet \bullet$ & $\bullet \bullet \bullet \bullet \bullet$ & $\bullet \bullet \bullet \bullet \bullet$ \\
\hline mono chrome & $\bullet \bullet \circ$ & 00000 & 00000 \\
\hline omnidirectional & 000 & 00000 & $\bullet \circ 000$ \\
\hline special lens & $\bullet \circ$ & $\bullet ० 000$ & $\bullet$ \\
\hline Laser scanner & $\bullet \circ$ & 00000 & $\bullet \bullet \bullet ० \circ$ \\
\hline $3 \mathrm{D}$ & $\bullet$ & 00000 & $\bullet \circ 000$ \\
\hline Acceleration & $\bullet$ & $\bullet \circ 000$ & $\bullet \bullet \bullet \bullet \circ$ \\
\hline Gyroscope & $\bullet \bullet$ & $\bullet 0000$ & $\bullet \bullet \bullet \bullet$ \\
\hline
\end{tabular}

frame rate during their presentations. Other special configurations like omnidirectional cameras or special image generation or wide angle lenses are used by single teams. Individual teams integrated a laser scanner or a 3D sensor. Both approaches seem to be promising [10] but increase the additional integration effort as well.

Inertial measurements are able to improve position estimations significantly. It is interesting to note, that the majority of the Yellow group applies gyroscopes and acceleration sensors. But their use is limited in the more successful groups.

i) Hardware platforms: The different tasks - environment perception, measurement and image processing, trajectory planning, actuator control, etc. - are implemented on a multi-controller system by all teams. We found various combinations of different powerful PC boards with low performance 8 or 32 Bit processors used as motor controllers or sensor interfaces. Most competitors of the Carolo-Cup 2014 used a configuration with 3 controllers Fig. 4c.

Tailored electronic components like motor drivers are desirable in robotic projects. Compared to standard components they provide task specific performance and dimensions combined with an optimized energy consumption. But the development process ties up a lot of resources and involves a huge number of pitfalls. Against this background, it is notable that less successful teams mostly decided to develop individual boards. In contrast to this decision, the number 
of students from electrical engineering is smaller in this group than in the others, as visible in Tab. 3a. The same pattern is visible for brushless motors. They provide a number of benefits but require a more complex driver unit. Again, the teams from the group Yellow integrated the more challenging way.

The construction kits for car models do not meet the minimum wheelbase covered by the competition rules. In order to achieve a benefit for the parking discipline some teams shortens the chassis. One team has used a rear steering axle in addition to the front steering axle. The team has used this steering geometry for all three competitions. In the competitions lane-following and overtaking obstacles they have used the rear steering axle for driving faster in the curves. In the parking competition, the team used the steering geometry for crab-motion like parking by driving forward and backward.

Table IV

HARDWARE CONFIGURATIONS

\begin{tabular}{|l|c|c|c|}
\cline { 2 - 4 } \multicolumn{1}{c|}{} & \multicolumn{3}{c|}{ Group } \\
\hline Parameter & Green & Blue & Yellow \\
\hline \hline Self-designed boards & $\bullet \bullet \circ$ & $\bullet \circ 000$ & $\bullet \bullet \bullet \bullet \circ$ \\
\hline Shortened chassis & $\bullet \bullet \circ$ & $\bullet \bullet 000$ & 00000 \\
\hline Steering rear axle & $\bullet \circ 0$ & 00000 & $\bullet \bullet 000$ \\
\hline Brushless motors & $\circ 00$ & $\bullet \bullet 000$ & $\bullet \bullet \bullet \bullet \circ$ \\
\hline
\end{tabular}

j) Software platforms: It is noticeable that two of the three teams in the group Green uses ADTF (Automotive Data and Time Triggered Framework), ADTF brings a built-in logging and realtime data recording and playback with, what debugging simplifies enormously since errors so easily can be reproduced. Furthermore, modularization is given by the concept of ADTF, which further simplifies the development. Because of these functionalities remains more time for other tasks.

Table V

SOFTWARE LIBRARIES AND OPERATING SYSTEMS IN USE.

\begin{tabular}{|c|c|c|c|}
\hline & \multicolumn{3}{|c|}{ Group } \\
\hline Parameter & Green & Blue & Yellow \\
\hline Windows & $\bullet$ & .0000 & 00000 \\
\hline Linux & $\bullet \bullet$ & $\bullet \bullet \bullet ० \circ$ & $\bullet \bullet \bullet ० \circ$ \\
\hline others & 000 & $\bullet$ & 00000 \\
\hline ADTF & $\bullet \bullet$ & 00000 & 00000 \\
\hline QT & $\bullet \bullet$ & $\bullet ० 000$ & $\bullet 0000$ \\
\hline OpenCV & $\bullet \bullet$ & $\bullet \bullet \bullet \bullet ०$ & $\bullet \bullet \bullet \bullet \bullet$ \\
\hline OSG & $\bullet \circ$ & 00000 & 00000 \\
\hline Boost & $\bullet$ & $\bullet \bullet ० ० ०$ & $\bullet \circ 000$ \\
\hline openNI & $\bullet$ & 00000 & $\bullet 0000$ \\
\hline armadillo & $\bullet$ & 00000 & 00000 \\
\hline OpenDaVINCI & 000 & 00000 & $\bullet 0000$ \\
\hline dlib & 000 & 00000 & $\bullet 0000$ \\
\hline Erlang-ALE & 000 & 00000 & $\bullet ० 000$ \\
\hline Erlang-NIF & 000 & 00000 & $\bullet 0000$ \\
\hline
\end{tabular}

Most teams use OpenCV for image processing, but OpenCV is mainly optimized for $\mathrm{x} 86$ systems, so the teams with $\mathrm{x} 86$ systems profited from the improvements most. Also it can be seen that the only team in the group Green which uses an ARM processor does not use OpenCV.

Almost all teams use Linux as operating system which results in a free choice of processors. Linux also has the advantage, that it can be largely adapted to the requirements. That one team in the group Green uses Windows, shows that the selection of the operating system is not critical for success.
Table VI shows the development priorities shown in the respective presentations. It is easy to see that the team in Green that does not use ADTF, has spent a lot of work in logging, simulation and modularity. Through its development of their own framework, the team was able to consider real-time conditions. However, also teams in the Blue and Yellow group has observed real-time conditions. That shows that real-time capability are not absolutely necessary for success .

Table VI

DEVELOPMENT FOCUS

\begin{tabular}{|c|c|c|c|}
\hline & \multicolumn{3}{|c|}{ Group } \\
\hline Parameter & Green & Blue & Yellow \\
\hline Real-time & $\bullet$ & $\bullet ০ ০ ০ ০$ & $\bullet ০ ০ ০ ০$ \\
\hline Image processing & 000 & $\bullet \bullet \bullet ০ ০$ & $\bullet \bullet ০ ০ ০$ \\
\hline Mapping & $\bullet$ & 00000 & $\bullet \circ ০ ০ ০$ \\
\hline Modularity & $\bullet$ & $\bullet \bullet ০ ০ ০$ & ০০০০০ \\
\hline Scheduling & $\bullet$ & $\bullet \circ \circ \circ \circ$ & $\bullet 0000$ \\
\hline Fault-tolerance & $\bullet \bullet$ & 00000 & $\bullet \circ ০ ০ ০$ \\
\hline Simulation & $\bullet \circ ০$ & $\bullet 0000$ & $\bullet \bullet \circ ০ ০$ \\
\hline Logging & $\bullet$ & 00000 & 00000 \\
\hline
\end{tabular}

\section{LESSONS LEARNED}

To address $R Q-3$, we present and discuss here the lessons learned from our teams. Therefore, we ran a retrospective meeting with our teams after returning from the competition to collect their impressions and suggestions.

\section{A. General and Organizational Lessons Learned}

Foremost, it is important to have team members who are committed to the common goal of realizing a self-driving miniature car that can participate in the student competition. The team's knowledge needs to cover mechanical, electrical, and software engineering skills.

At the beginning, it is important to study thoroughly the rules and regulations document to get to know what is precisely required for avoiding late minute additions to the car. For all disciplines, prefer simple and robust approaches over complicated ones; any approach must be mastered and the students need to know where to tune if the self-driving miniature car is not behaving as expected.

\section{B. Planning Lessons Learned}

In general, the endeavor of participating in the Carolo-Cup competition should be started as early as possible because hardware purchasing processes and team formation usually take time. For the project's milestone planning, define early milestones with an iterative development and integration process. In addition, a rotating role of a team manager was suggested.

Furthermore, the students shall present more and more realized functional features on the miniature car at all milestones preferably on a monthly basis. Finally, having a well-structured and good presentation is very important as more than one third of the total points can be achieved in this discipline.

\section{Conceptual Lessons Learned}

From the conceptual point of view, it is very important to not always start over each year; instead, focus on improvements of an existing hardware and software platform. Thus, the students can focus more on the algorithmic approaches to handle aspects where a previous year's team was not successful so far.

The algorithms for the different driving tasks should be evaluated early in a simulation environment to identify conceptual flaws. 
However, once a running algorithm was developed, it should be transferred to the target platform to identify performance gaps and data processing bottlenecks. Finally, all software components need to be integrated continuously with the hardware to enable early testing on the test track.

\section{Hardware Lessons Learned}

Designing competitive algorithms is already a big challenge in this competition; however, realizing them in a robust and reliable manner on a hardware environment is also pretty difficult. The main reason is that hardware components are on the critical project part as they need to be ordered and delivered; furthermore, low-cost components can easily break when they are not handled with care.

Therefore, it is important to start the ordering processes as early as possible. Furthermore, replacement parts shall be ordered as well so that a team can assemble in the best case two miniature cars. For this purpose, students with mechatronics, electrical, and mechanical background need to be involved to avoid searching for software bugs where, for instance, an unstable power supply might be the root-cause of an unexpected behavior of the car.

While printed circuit boards (PCB) enable a very use-case specific realization of the hardware interface, their assembly is errorprone, tedious, and time-consuming. To have a high quality for such software/hardware interface boards, it is recommended to use commercial-of-the-shelf (COTS) components as they are usually cheap and easily replaceable in case of hardware faults.

Since having a stable and robust image processing algorithm is important for a good performance at the competition, a fast embedded system to process the image data stream is recommended. However, potential camera driver issues need to be kept in mind as the camera supplier might not have specific drivers for the embedded board in use. This applies also to other sensors as well, while it is advised to limit the number of sensors and to focus on components with software/hardware interfaces that are easy to handle.

Finally, the development and maintenance process for the miniature must not rely on a Wifi connection as the competition arena might be congested. Thus, stable and fast connections to the car might be difficult to realize. In this regard, a good debugging interface and data logging layer is also helpful to understand why the miniature car did not behave as expected during a test or the competition.

\section{FUTURE WORK}

In this paper, we have summarized the 2014 edition of the annual student competition Carolo-Cup for self-driving miniature cars. The goal for the contestants is to realize a 1:10 scale vehicle that is able to following lane-markings, overtake obstacles made out of paper boxes, behave correctly at intersections, and park as quickly as possible on a sideways parking strip. Furthermore, the students need to present their technical concepts to a jury consisting of industrial practitioners and researchers.

We analyzed the teams' presentations given in this year's competition to present technical approaches and solution strategies. In detailed tables, we presented the results as a guidance for future contestants. Furthermore, we reported about the lessons learned from two different teams as gathered in a retrospective session right after the competition.

Our main findings are that a winning team needs to focus on a stable platform both for the software and the hardware architecture. Thus, a team can improve certain aspects of an algorithm for the next year's competition. Furthermore, hardware components, their purchase, delivery, and assembling process is considered to be crucial for a team's success. Starting early with few components that can be easily mastered in terms of integration and maintenance is very important to not delay the testing time on the test track.

These findings are also confirmed by the winner of the 2005 DARPA Grand Challenge S. Thrun who confirms that "software is the key to robotic driving" [11]. In this regards, future work for the Carolo-Cup competition could also include the definition of a standardized hardware platform so that newcomer teams can easily enter the contest in a newly established league for instance. Having such a platform could also enable new driving tasks like cooperative maneuvers among several self-driving cars, which are currently not addressed in the competition.

\section{ACKNOWLEDGMENT}

This work was partially supported by the EU under the FP7-ICT programme, through project 288195 "Kernel-based ARchitecture for safetY-critical cONtrol” (KARYON).

\section{REFERENCES}

[1] R. D. Beer, H. J. Chiel, and R. F. Drushel, "Using autonomous robotics to teach science and engineering," Communications of the ACM, vol. 42, no. 6, pp. 85-92, 1999.

[2] T. Niemueller, D. Ewert, S. Reuter, A. Ferrein, S. Jeschke, and G. Lakemeyer, "Robocup logistics league sponsored by festo: a competitive factory automation testbed," in Proc. of the RoboCup Symposium, 2013.

[3] N. Chen, "A vision-guided autonomous vehicle: an alternative micromouse competition," Education, IEEE Transactions on, vol. 40, no. 4, pp. 253-258, Nov. 1997.

[4] S. Kibler, A. Hauer, D. Giessel, C. Malveaux, and D. Raskovic, "Ieee micromouse for mechatronics research and education," in Mechatronics (ICM), 2011 IEEE International Conference on, Apr. 2011, pp. 887-892.

[5] T. Bräunl, "Research relevance of mobile robot competitions," IEEE Robotics \& Automation Magazine, vol. 6, no. 4, pp. 3237, 1999.

[6] C. Berger, "From a Competition for Self-Driving Miniature Cars to a Standardized Experimental Platform: Concept, Models, Architecture, and Evaluation," Journal of Software Engineering for Robotics, vol. 5, no. 1, pp. 63-79, May 2014.

[7] _ - "Educational Effect of a Student Competition with SelfDriving Cars on Selected Software Engineering Topics," 2013.

[8] F. W. Rauskolb, K. Berger, C. Lipski, M. Magnor, K. Cornelsen, J. Effertz, T. Form, F. Graefe, S. Ohl, W. Schumacher, J.-M. Wille, P. Hecker, T. Nothdurft, M. Doering, K. Homeier, J. Morgenroth, L. Wolf, C. Basarke, C. Berger, T. Gülke, F. Klose, and B. Rumpe, "Caroline: An Autonomously Driving Vehicle for Urban Environments," Journal of Field Robotics, vol. 25, no. 9, pp. 674-724, Sep. 2008. [Online]. Available: http://dx.doi.org/10.1002/rob.20254.

[9] J. Hirsch, Self-driving cars inch closer to mainstream availability, Oct. 2013. [Online]. Available: http://www.latimes. com/business/autos/la-fi-adv-hy-self-driving-cars-20131013, 0,5094627.story (visited on 10/15/2013).

[10] S. Zug, F. Penzlin, A. Dietrich, T. T. Nguyen, and S. Albert, "Are Laser Scanners Replaceable by Kinect Sensors in Robotic Applications?" In IEEE International Symposium on Robotic and Sensors Environments (ROSE 2012), Magdeburg, Germany, 2012.

[11] S. Thrun, "Toward Robotic Cars," Commununications of the ACM, vol. 53, no. 4, pp. 99-106, Apr. 2010. 